\title{
Evaluation of a Modified Reynolds Stress Model for Turbulent Dispersed Two-Phase Flows Including Two-Way Coupling
}

\author{
N. A. Beishuizen · B. Naud · D. Roekaerts
}

Received: 13 December 2006 / Accepted: 25 February 2007 /

Published online: 28 July 2007

(C) Springer Science + Business Media B.V. 2007

\begin{abstract}
A modified Reynolds stress turbulence model for the pressure rate of strain can be derived for dispersed two-phase flows taking into account gas-particle interaction. The transport equations for the Reynolds stresses as well as the equation for the fluctuating pressure can be derived starting from the multiphase NavierStokes equations. The unknown pressure rate of strain correlation in the Reynolds stress equations is then modelled by considering the multiphase equation for the fluctuating pressure. This leads to a multiphase pressure rate of strain model. The extra particle interaction source terms occurring in the model for the pressure rate of strain can be constructed easily, with no noticeable extra computational cost. Eulerian-Lagrangian simulation results of a turbulent dispersed two-phase jet are presented to show the differences in results with and without the new two-way coupling terms.
\end{abstract}

Keywords Turbulence • Two-way coupling • Dispersion model • Sprays • Multiphase flows

\section{Introduction}

In turbulent dispersed two-phase flows, the dispersed phase introduces a force acting on the carrier medium. This force appears as a source term in the Navier-Stokes equations. From the exact multiphase Navier-Stokes equations [2, 8, 16], the mean momentum, Reynolds stress and dissipation equations can be derived. The terms describing the effects of the dispersed phase on the gas phase in these equations are

\footnotetext{
N. A. Beishuizen $(\bowtie) \cdot$ D. Roekaerts

Department of Multi-Scale Physics, Delft University of Technology, Lorentzweg 1, 2628 CJ Delft, The Netherlands e-mail: n.a.beishuizen@tudelft.nl

B. Naud

Modelling and Numerical Simulation Group, Energy Department, Ciemat, Avda. Complutense 22, 28040 Madrid, Spain
} 
referred to as two-way coupling terms. The two-way coupling terms can be evaluated by considering the jump condition at the interfaces $[6,9]$. Several models for the source terms appearing in the Reynolds stress equation can be found in the literature $[1,6,9]$. The main difference lies in the treatment of the velocity fluctuation at the interface of a particle. Moreover, a model for the unknown pressure rate of strain $\Pi_{i j}$ can be found by using the equation for the fluctuating pressure, which can also be derived from the multiphase Navier-Stokes equations. A modified expression for the pressure rate of strain taking into account the presence of the dispersed phase has been derived first by Taulbee et al. [11, 22] following the approach of Launder et al. [10] to obtain an extension of the LRR Reynolds stress model. Following the same approach as Speziale et al. [21], we obtain an extension of the SSG model. From this more general formalism, the dispersed-phase LRR model of [11, 22] can be recovered.

Although the two-way coupling terms appearing explicitly in the momentum and Reynolds stress transport equations are often taken into account in two-phase flow simulations, the model for the modified pressure rate of strain $\Pi_{i j}^{(\mathrm{fp})}$ is new and the two-way coupling terms appearing in $\Pi_{i j}$ were not previously taken into account in simulations of dispersed multiphase flows.

In the equation of motion for the dispersed phase, the main contribution is the drag term, which has the form $m_{\mathrm{p}}\left(U_{\mathrm{s}, i}-U_{\mathrm{p}, i}\right) / \tau_{\mathrm{p}}$, where $\tau_{\mathrm{p}}$ is the particle relaxation time and $\boldsymbol{U}_{\mathrm{s}}$ is the instantaneous undisturbed fluid flow velocity at the location of the particle $[5,12]$. This velocity 'seen' by the particles is an unknown in RANS and PDF approaches. In this work, we use the dispersion model based on the solution of a Langevin equation, described by Simonin et al. [19] and Minier and Peirano [13] to model $\boldsymbol{U}_{\mathrm{s}}$.

In this paper we will focus on discussing the new pressure rate of strain model and its performance versus a traditional approach. In the next section we will give the governing equations for the continuous phase and the dispersed phase. Then, the continuous phase modelling approach will be discussed. The emphasis lies on the model for the pressure rate of strain. The dispersed phase modelling approach is discussed briefly and the effects of adding these extra terms are then investigated numerically using a polydispersed particle laden jet. This same jet was investigated experimentally by Ferrand et al. [3]. To our knowledge this experiment is unique in the sense that it combines the complexity of polydispersity and two-way coupling. This polydispersed particle laden jet was also chosen in Naud [16] to investigate the behaviour of the particle dispersion model. Other experiments possibly of interest for model validation exist. A well documented polydispersed spray experiment is the vaporising spray of Sommerfeld and Qiu [20]. However, no strong two-way coupling is present in this spray. A monodispersed particle laden jet was investigated experimentally by Hishida and Maeda [7], and a monodispersed particle laden flow has also been investigated by Mostafa et al. [14].

The mean gas-phase properties are obtained in an Eulerian way using a Reynolds averaged Navier-Stokes (RANS) solver. For the dispersed phase, we model and solve the dispersed phase transport equation for the mass density function (MDF). We do this in a Lagrangian way by using a Monte Carlo method to solve the equations of motion for the dispersed phase. The unknown model constants that appear in the extra terms for the pressure strain rate are taken from the DNS results of Taulbee et al. [22]. Since no information on the value of the constant in the SSG 
term is available, this constant will be set to zero. The model used then reduces to the multiphase LRR model.

\section{Governing Equations}

\subsection{Instantaneous Eulerian equations for the continuous phase}

The instantaneous continuity and momentum equations for the continuous phase of a dispersed two-phase flow (without mass transfer) read $[2,8,16]$ :

$$
\begin{aligned}
\frac{\partial}{\partial t}(\rho \mathcal{X})+\frac{\partial}{\partial x_{k}}\left(\rho U_{k} \mathcal{X}\right) & =0 \\
\frac{\partial}{\partial t}\left(\rho U_{i} \mathcal{X}\right)+\frac{\partial}{\partial x_{k}}\left(\rho U_{i} U_{k} \mathcal{X}\right) & =-\frac{\partial(p \mathcal{X})}{\partial x_{i}}+\frac{\partial\left(\tau_{i k} \mathcal{X}\right)}{\partial x_{k}}+(\rho \mathcal{X}) g_{i}+\mathcal{S}_{U_{i}},
\end{aligned}
$$

where $\mathcal{X}(\boldsymbol{x}, t)$ is the indicator function of the continuous phase: equal to one when the continuous phase is present at $(\boldsymbol{x}, t)$ and equal to zero otherwise. $\mathcal{S}_{U_{i}}$ is the twoway coupling source term: the source of momentum due to stress at the interface (surface forces). The Newtonian stress tensor in the continuous phase reads:

$$
\tau_{i j} \mathcal{X}=\mu \mathcal{X}\left[\left(\frac{\partial U_{i} \mathcal{X}}{\partial x_{j}}+\frac{\partial U_{j} \mathcal{X}}{\partial x_{i}}\right)-\frac{2}{3} \frac{\partial U_{k} \mathcal{X}}{\partial x_{k}} \delta_{i j}\right]
$$

\subsection{Lagrangian particle equation of motion}

Simplified equations of motion are used to describe the velocity evolution of a heavy particle with mass $m_{\mathrm{p}}$ in a turbulent fluid [5, 12]:

$$
\begin{aligned}
\frac{\mathrm{d} X_{\mathrm{p}, i}}{\mathrm{~d} t} & =U_{\mathrm{p}, i}, \\
m_{\mathrm{p}} \frac{\mathrm{d} U_{\mathrm{p}, i}}{\mathrm{~d} t} & =m_{\mathrm{p}} \frac{U_{\mathrm{s}, i}-U_{\mathrm{p}, i}}{\tau_{\mathrm{p}}}-\frac{m_{\mathrm{p}}}{\rho_{\mathrm{p}}} \frac{\partial\langle p\rangle}{\partial x_{i}}+m_{\mathrm{p}} g_{i} .
\end{aligned}
$$

The effect of the surrounding fluid flow is included through the first two terms on the right hand side of (5), respectively the drag force and the mean pressure gradient at the particle location. The seen velocity $\boldsymbol{U}_{\mathrm{s}}$ is the velocity of the undisturbed fluid flow at the position of the particle centre-the velocity that would exist in the absence of the particle but turbulent and disturbed by all the other particles [18].

The particle response time scale $\tau_{\mathrm{p}}$ is given by the response time in a Stokes regime $\tau_{\mathrm{p}}^{(\mathrm{St})}$ :

$$
\frac{1}{\tau_{\mathrm{p}}}=\frac{f_{1}}{\tau_{\mathrm{p}}^{(\mathrm{St})}} \quad \text { with } \quad \tau_{\mathrm{p}}^{(\mathrm{St})}=\frac{\rho_{\mathrm{p}} D_{p}^{2}}{18 \mu_{\mathrm{f}}},
$$

where $f_{1}$ is the Schiller-Naumann correction for high Reynolds number flows:

$$
f_{1}=\left\{\begin{array}{ll}
1+0.15 R e_{\mathrm{p}}^{0.687} & \text { if } R e_{\mathrm{p}} \leq 1,000 \\
0.44 \frac{R e_{\mathrm{p}}}{24} & \text { if } R e_{\mathrm{p}}>1,000
\end{array} \text { with } R e_{\mathrm{p}}=\frac{\rho_{\mathrm{f}}\left|\boldsymbol{U}_{\mathrm{p}}-\boldsymbol{U}_{\mathrm{s}}\right| D_{\mathrm{p}}}{\mu_{\mathrm{f}}}\right.
$$




\section{Modelling of the Continuous Phase}

\subsection{Mean equations}

The mean continuity and mean momentum equations are obtained by taking the expected value \langle\rangle of (1) and (2):

$$
\begin{gathered}
\frac{\partial \bar{\rho}}{\partial t}+\frac{\partial \bar{\rho} \widetilde{U}_{k}}{\partial x_{k}}=0 \\
\bar{\rho} \frac{\partial \widetilde{U}_{i}}{\partial t}+\bar{\rho} \widetilde{U}_{k} \frac{\partial \widetilde{U}_{i}}{\partial x_{k}}=-\frac{\partial \bar{\rho} \widetilde{u}_{i}^{\prime \prime} u_{k}^{\prime \prime}}{\partial x_{k}}-\frac{\partial \bar{p}}{\partial x_{i}}+\frac{\partial \overline{\tau_{i k}}}{\partial x_{k}}+\bar{\rho} g_{i}+\left\langle\mathcal{S}_{U_{i}}\right\rangle,
\end{gathered}
$$

where the following notations have been introduced:

$$
\bar{Q}=\langle Q \mathcal{X}\rangle \quad \text { and } \quad \widetilde{Q}=\frac{\langle\rho Q \mathcal{X}\rangle}{\langle\rho \mathcal{X}\rangle}
$$

To be more precise, the notation \langle\rangle refers to the expected value of volume averages over small volumes.

Considering that the integral of the forces exerted on a droplet surface are equal to the drag force, the mean source term $\left\langle\mathcal{S}_{U_{i}}\right\rangle$ describing momentum transfer at the particle interface is obtained as a sum over the droplets present in a small domain $\Omega$ of volume $\mathcal{V}_{\Omega}$ :

$$
\left\langle\mathcal{S}_{U_{i}}\right\rangle=-\frac{1}{\mathcal{V}_{\Omega}}\left\langle\sum_{\Omega} m_{\mathrm{p}}\left(\frac{\mathrm{d} U_{\mathrm{p}, i}}{\mathrm{~d} t}-\frac{1}{\rho_{\mathrm{p}}} \frac{\partial\langle p\rangle}{\partial x_{i}}-g_{i}\right)\right\rangle .
$$

Note that the notations introduced in (10) imply that the mean density and pressure appearing in (8) and (9) are averaged over the whole two-phase flow. For instance, in case of a constant density $\rho_{\mathrm{f}}$ (as is the case for the simulations presented in a subsequent section):

$$
\bar{\rho}=\langle\mathcal{X}\rangle \rho_{\mathrm{f}}
$$

where $\langle\mathcal{X}\rangle$ is the probability of presence of the continuous phase (i.e. the volume fraction of the continuous phase since we consider volume averages).

\subsection{Reynolds stress transport equation}

From the fluctuating velocity transport equation:

$$
\begin{aligned}
& \frac{\partial}{\partial t}\left(\rho u_{i}^{\prime \prime} \mathcal{X}\right)+\frac{\partial}{\partial x_{k}}\left(\rho u_{i}^{\prime \prime} \widetilde{U}_{k} \mathcal{X}\right)+\rho u_{k}^{\prime \prime} \mathcal{X} \frac{\partial \widetilde{U}_{i}}{\partial x_{k}} \\
& \quad=-\frac{\partial p \mathcal{X}}{\partial x_{i}}+\frac{\partial \tau_{i k} \mathcal{X}}{\partial x_{k}}-\frac{\partial}{\partial x_{k}}\left(\rho u_{i}^{\prime \prime} u_{k}^{\prime \prime} \mathcal{X}\right)+\mathcal{S}_{U_{i}}-\rho \mathcal{X}\left[\frac{\partial \widetilde{U}_{i}}{\partial t}+\widetilde{U}_{k} \frac{\partial \widetilde{U}_{i}}{\partial x_{k}}-g_{i}\right],
\end{aligned}
$$


we obtain the exact Reynolds stress transport equation:

$$
\begin{aligned}
& \frac{\partial}{\partial t}\left(\bar{\rho} \widetilde{u}_{i}^{\pi \prime} u_{j}^{\prime \prime}\right)+\frac{\partial}{\partial x_{k}}\left(\bar{\rho} \widetilde{u}_{i}^{\prime \prime} u_{j}^{\prime \prime} \widetilde{U}_{k}\right)+\bar{\rho} u_{i}^{\prime \prime} u_{k}^{\prime \prime} \frac{\partial \widetilde{U}_{j}}{\partial x_{k}}+\bar{\rho} u_{j}^{\pi \prime} u_{k}^{\prime \prime} \frac{\partial \widetilde{U}_{i}}{\partial x_{k}} \\
& \quad=-\left[\overline{u_{i}^{\prime \prime} \frac{\partial p}{\partial x_{j}}}+\overline{u_{j}^{\prime \prime} \frac{\partial p}{\partial x_{i}}}\right]+\left[\overline{u_{i}^{\prime \prime} \frac{\partial \tau_{j k}}{\partial x_{k}}}+\overline{u_{j}^{\prime \prime} \frac{\partial \tau_{i k}}{\partial x_{k}}}\right]-\frac{\partial}{\partial x_{k}}\left[\bar{\rho} u_{i}^{\prime \prime \prime} u_{j}^{\prime \prime} u_{k}^{\prime \prime}\right]+\left\langle\mathcal{S}_{u_{i} u_{j}}\right\rangle,
\end{aligned}
$$

with

$$
\left\langle\mathcal{S}_{u_{i} u_{j}}\right\rangle=\left\langle u_{I, i}^{\prime \prime} \mathcal{S}_{U_{j}}\right\rangle+\left\langle u_{I, j}^{\prime \prime} \mathcal{S}_{U_{i}}\right\rangle,
$$

where $\boldsymbol{u}_{I}^{\prime \prime}$ is the velocity fluctuation at the interface. Its meaning will be discussed later.

Introducing the dissipation $\epsilon$ :

$$
\epsilon=\frac{1}{2} \epsilon_{k k} \quad \text { with } \quad \bar{\rho} \epsilon_{i j}=2\left\langle\mu \mathcal{X} \frac{\partial u_{i}^{\prime \prime} \mathcal{X}}{\partial x_{k}} \frac{\partial u_{j}^{\prime \prime} \mathcal{X}}{\partial x_{k}}\right\rangle,
$$

and neglecting the terms $\left\langle u_{i}^{\prime \prime} \mathcal{X}\right\rangle$ and $\partial\left(u_{k}^{\prime \prime} \mathcal{X}\right) / \partial x_{k}$, and the molecular diffusion terms, (14) can be written as:

$$
\frac{\partial}{\partial t}\left(\bar{\rho} \widetilde{u}_{i}^{\prime \prime} u_{j}^{\prime \prime}\right)+\frac{\partial}{\partial x_{k}}\left(\bar{\rho} \widetilde{u_{i}^{\prime \prime} u_{j}^{\prime \prime}} \widetilde{U}_{k}\right)=P_{i j}+\mathcal{T}_{i j}+\Pi_{i j}-\frac{2}{3} \bar{\rho} \epsilon \delta_{i j}+\left\langle\mathcal{S}_{u_{i} u_{j}}\right\rangle
$$

where

$$
\begin{aligned}
P_{i j} & =-\bar{\rho} u_{i}^{\prime \prime} u_{k}^{\prime \prime} \frac{\partial \widetilde{U}_{j}}{\partial x_{k}}-\bar{\rho} u_{j}^{\prime \prime} u_{k}^{\prime \prime} \frac{\partial \widetilde{U}_{i}}{\partial x_{k}}, \\
\mathcal{T}_{i j} & =-\frac{\partial}{\partial x_{k}}\left[\bar{\rho} u_{i}^{\prime \prime} u_{j}^{\prime \prime} u_{k}^{\prime \prime}-\overline{p^{\prime} u_{i}^{\prime \prime}} \delta_{j k}-\overline{p^{\prime} u_{j}^{\prime \prime}} \delta_{i k}\right], \\
\Pi_{i j} & =\left\langle p^{\prime} \mathcal{X}\left(\frac{\partial u_{i}^{\prime \prime} \mathcal{X}}{\partial x_{j}}+\frac{\partial u_{j}^{\prime \prime} \mathcal{X}}{\partial x_{i}}\right)\right\rangle-\bar{\rho}\left(\epsilon_{i j}-\frac{1}{3} \epsilon_{k k} \delta_{i j}\right) .
\end{aligned}
$$

Under the assumption of local isotropy, the last term in (20) cancels, and $\Pi_{i j}$ is the pressure rate of strain.

\subsection{Pressure rate of strain model}

The trace of $\Pi_{i j}$ is zero in constant density flows (it is a redistribution term), and therefore its modelling is not an issue when using $k-\epsilon$ type models. Models for the pressure rate of strain tensor $\Pi_{i j}$ can be constructed by examining the equation for the fluctuating pressure. Following the approach of Speziale et al. [21] to derive the SSG model, the pressure rate of strain takes the form:

$$
\Pi_{i j}=\Pi_{i j}^{(1)}+\Pi_{i j}^{(2)}
$$


where the slow part of the pressure rate of strain $\Pi_{i j}^{(1)}$ is modelled as:

$$
\Pi_{i j}^{(1)}=\bar{\rho} C_{1} \epsilon b_{i j}+\bar{\rho} C_{2} \epsilon\left(b_{i j}^{2}-\frac{1}{3} b_{k k}^{2} \delta_{i j}\right)
$$

and the rapid part $\Pi_{i j}^{(2)}$ :

$$
\begin{aligned}
\Pi_{i j}^{(2)}= & \bar{\rho} C_{3} k S_{i j}+\bar{\rho} C_{4} k\left(b_{i k} S_{j k}+b_{j k} S_{i k}-\frac{2}{3} b_{m n} S_{m n} \delta_{i j}\right) \\
& +\bar{\rho} C_{5} k\left(b_{i k} \Omega_{j k}+b_{j k} \Omega_{i k}\right)
\end{aligned}
$$

with $k=\widetilde{u_{k}^{\prime \prime}} u_{k}^{\prime \prime} / 2$ the turbulent kinetic energy, and with the mean strain rate tensor $S_{i j}$ and the mean vorticity tensor $\Omega_{i j}$ defined as:

$$
S_{i j}=\frac{1}{2}\left(\frac{\partial \tilde{U}_{i}}{\partial x_{j}}+\frac{\partial \tilde{U}_{j}}{\partial x_{i}}\right) \quad \text { and } \quad \Omega_{i j}=\frac{1}{2}\left(\frac{\partial \tilde{U}_{i}}{\partial x_{j}}-\frac{\partial \widetilde{U}_{j}}{\partial x_{i}}\right)
$$

The normalised fluid anisotropy tensor $b_{i j}$ is given by

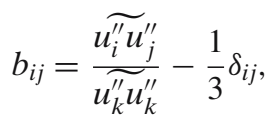

and the notation $b_{i j}^{2}$ is the usual abbreviation for $b_{i l} b_{l j}$. The model constants can be chosen in such a way that they correspond to well known turbulence models. The values of the constants for the Rotta model, the model of Launder, Reece and Rodi (LRR) [10] and the model of Speziale, Sarkar and Gatski (SSG) [21] are summarised in Table 1.

In order to derive a similar model for a dispersed two-phase flow, let's consider a situation of homogeneous turbulence without mean velocity gradient, for a constant density continuous phase, with a statistically uniform distribution of the dispersed phase. In this case, the Poisson equation for the fluctuating pressure $p^{\prime}$ can be obtained from the divergence of (13):

$$
-\frac{\partial^{2} p^{\prime} \mathcal{X}}{\partial x_{i} x_{i}}=\frac{\partial}{\partial x_{i}}\left[\frac{\partial \rho \mathcal{X} u_{i}^{\prime \prime} u_{k}^{\prime \prime}}{\partial x_{k}}\right]+\frac{\partial \mathcal{S}_{U_{i}}^{(I)}}{\partial x_{i}} .
$$

Table 1 Model constants for three different Reynolds stress models

\begin{tabular}{lllllll}
\hline Model & $C_{1}$ & $C_{2}$ & $C_{3}$ & $C_{4}$ & $C_{5}$ & Model constants \\
\hline Rotta & $-2 c_{1}$ & 0 & 0 & 0 & 0 & $c_{1}=4.15$ \\
LRR & $-2 c_{1}$ & 0 & $\frac{4}{3} c_{2}$ & $2 c_{2}$ & $2 c_{2}$ & $c_{1}=1.8, c_{2}=0.6$ \\
SSG & $-2 c_{1}-c_{1}^{\star} \frac{P}{\epsilon}$ & $c_{2}$ & $c_{3}-c_{3}^{\star} \sqrt{b_{i i}^{2}}$ & $c_{4}$ & $c_{5}$ & $c_{1}=1.7, c_{2}=4.2, c_{3}=0.8$ \\
& & & & & & $c_{4}=1.25, c_{5}=0.40$ \\
& & & & & & $c_{1}^{\star}=1.8, c_{3}^{\star}=1.30$ \\
\hline
\end{tabular}


This equation suggests that the two-way coupling source term plays a role in the return to isotropy in the slow part of the pressure strain correlation $\Pi_{i j}^{(1)}$. We propose to add to the slow part given by (22) a two-phase contribution $\Pi_{i j}^{(\mathrm{fp})}$ of the form:

$$
\Pi_{i j}^{(\mathrm{fp})}=-\frac{1}{2}\left\langle\mathcal{S}_{u_{k} u_{k}}\right\rangle\left[C_{1}^{(\mathrm{fp})} b_{i j}^{(\mathrm{fp})}+C_{2}^{(\mathrm{fp})}\left(b_{i j}^{2(\mathrm{fp})}-\frac{1}{3} b_{k k}^{2(\mathrm{fp})} \delta_{i j}\right)\right],
$$

with $b_{i j}^{(\mathrm{fp})}$ the normalised anisotropy tensor based on the Reynolds stress two-way coupling source terms:

$$
b_{i j}^{(\mathrm{fp})}=\frac{\left\langle\mathcal{S}_{u_{i} u_{j}}\right\rangle}{\left\langle\mathcal{S}_{u_{k} u_{k}}\right\rangle}-\frac{1}{3} \delta_{i j} .
$$

The factor $\frac{1}{2}\left\langle\mathcal{S}_{u_{k} u_{k}}\right\rangle$ in (27) specifies the time scale for the return to isotropy implied by $\Pi_{i j}^{(\mathrm{fp})}$, as we will see in (30). It plays the same role as the factor $\bar{\rho} \epsilon$ in (22). It is indeed the two-way coupling source term appearing in the turbulent kinetic transport equation next to $\bar{\rho} \epsilon$ (with opposite sign).

Mashayek et al. $[11,22]$ proposed the form for $\Pi_{i j}^{(\mathrm{fp})}$ corresponding to the LRR model: 1

$$
\Pi_{i j}^{(\mathrm{fp})}=-C_{f 3}\left\langle\mathcal{S}_{u_{k} u_{k}}\right) b_{i j}^{(\mathrm{fp})}
$$

with $C_{f_{3}}=\frac{1}{2} C_{1}^{(\mathrm{fp})}$. In the homogenous situation considered to obtain (26), this model implies the following evolution for the anisotropy tensor $b_{i j}$ :

$$
\frac{\mathrm{d} b_{i j}}{\mathrm{~d} t}=\left(2-C_{1}\right) \omega b_{i j}+2 \omega^{(f p)}\left[\frac{\left\langle\mathcal{S}_{u_{i} u_{j}}\right\rangle}{\left\langle\mathcal{S}_{u_{k} u_{k}}\right\rangle}-\frac{\widetilde{u_{i}^{\prime \prime} u_{j}^{\prime \prime}}}{u_{k}^{\prime \prime} u_{k}^{\prime \prime}}-C_{f 3} b_{i j}^{(\mathrm{fp})}\right],
$$

with

$$
\omega=\frac{2 \epsilon}{u_{k}^{\prime \prime} u_{k}^{\prime \prime}} \quad \text { and } \quad \omega^{(f p)}=\frac{\left\langle\mathcal{S}_{u_{k} u_{k}}\right\rangle}{\bar{\rho} u_{k}^{\prime \prime} u_{k}^{\prime \prime}} .
$$

The first term on the right hand side of (30) is the linear model of Rotta where the time scale of return to isotropy is $1 / \omega$. The last term represents the effect of two-way coupling on the return to isotropy. The time scale $1 / \omega^{(\mathrm{fp})}$ is based on the two-way coupling source term appearing in the turbulent kinetic energy transport equation.

When extending the SSG turbulence model to dispersed two-phase flows, only two extra constants need to be evaluated. Mashayek and Taulbee [11] obtained a value of $C_{f 3}=0.5$ (or $C_{1}^{(\mathrm{fp})}=1$ ) from their direct numerical simulations. No information is available on the model constant $C_{2}^{(\mathrm{fp})}$. Therefore, we will now restrict to the model (29) proposed by Mashayek et al. [11], in the context of Rotta or LRR Reynolds stress models.

\footnotetext{
${ }^{1}$ Note that in their Eulerian-Eulerian formulation Mashayek and Taulbee do not introduce the source term $\mathcal{S}_{U_{i}}$ appearing in (2). Instead, they directly express mean source terms as functions of volume fraction, $\tau_{\mathrm{p}}$, and particle and fluid velocity difference by considering the drag force for a monodispersed spray.
} 
3.4 Reynolds-stress modelled equations

Using a generalised gradient diffusion model for the turbulent flux $\mathcal{T}_{i j}$, the modelled Reynolds stress equation reads:

$$
\begin{aligned}
\frac{\partial \bar{\rho} \tilde{u}_{i}^{\prime \prime} u_{j}^{\prime \prime}}{\partial t}+\frac{\partial \bar{\rho} \widetilde{U}_{k} \widetilde{u}_{i}^{\prime \prime} u_{j}^{\prime \prime}}{\partial x_{k}}= & \frac{\partial}{\partial x_{k}}\left[\left(\bar{\mu} \delta_{k l}+\frac{C_{s}}{\omega} \bar{\rho} \widetilde{u}_{k}^{\prime \prime} u_{l}^{\prime \prime}\right) \frac{\partial \widetilde{u}_{i}^{\prime \prime} u_{j}^{\prime \prime}}{\partial x_{l}}\right]-\frac{2}{3} \bar{\rho} \epsilon \delta_{i j} \\
& +P_{i j}+\Pi_{i j}^{(1)}+\Pi_{i j}^{(2)}+\Pi_{i j}^{(\mathrm{fp})}+\left\langle\mathcal{S}_{u_{i} u_{j}}\right\rangle
\end{aligned}
$$

In the same way as for the mean momentum source term (11), the Reynolds stress two-way coupling source term $\left\langle\mathcal{S}_{u_{i} u_{j}}\right\rangle(15)$ is obtained from:

$$
\left\langle u_{I, i}^{\prime \prime} \mathcal{S}_{U_{j}}\right\rangle=-\frac{1}{\mathcal{V}_{\Omega}}\left\langle\sum_{\Omega} m_{\mathrm{p}} u_{I, i}^{\prime \prime}\left(\frac{\mathrm{d} U_{\mathrm{p}, j}}{\mathrm{~d} t}-\frac{1}{\rho_{\mathrm{p}}} \frac{\partial\langle p\rangle}{\partial x_{j}}-g_{j}\right)\right\rangle .
$$

The velocity of the fluid phase on the particle surface is assumed to be equal to the velocity of the particle. The fluid velocity fluctuation at the interface is then obtained as:

$$
u_{I, i}^{\prime \prime}=U_{\mathrm{p}, i}-\tilde{U}_{i}
$$

This approach corresponds to the 'Consistent Terms' mentioned in [9] and derived previously in [1], where it is claimed that wake-induced turbulence is taken into account. However, this does not correspond to the work of Mashayek and Taulbee where they assume that $u_{I, i}^{\prime \prime}$ is the fluid velocity fluctuation. Nevertheless, we will keep the value determined in [11] for the model constant $C_{1}^{(\mathrm{fp})}=1$.

\subsection{Dissipation modelled equation}

A formal derivation of the transport equation for dissipation leads to a two-way coupling source term of the form

$$
\left\langle\mathcal{S}_{\epsilon}\right\rangle=2\left\langle\mu \mathcal{X} \frac{\partial u_{i}^{\prime \prime} \mathcal{X}}{\partial x_{j}} \frac{\partial \mathcal{S}_{U_{i}}}{\partial x_{j}}\right\rangle
$$

This source term is usually modelled as

$$
\left\langle\mathcal{S}_{\epsilon}\right\rangle=\frac{C_{\epsilon_{3}} \epsilon}{u_{k}^{\prime \prime} u_{k}^{\prime \prime}}\left\langle\mathcal{S}_{u_{k} u_{k}}\right\rangle,
$$

where the value of the constant $C_{\epsilon_{3}}=1.8$ is taken from [9]. The following transport equation is solved:

$$
\begin{aligned}
\frac{\partial \bar{\rho} \epsilon}{\partial t}+\frac{\partial \bar{\rho} \widetilde{U}_{j} \epsilon}{\partial x_{j}}= & \frac{\partial}{\partial x_{j}}\left[\left(\bar{\mu} \delta_{j k}+\frac{C_{s}^{\epsilon}}{\omega} \bar{\rho} \widetilde{u}_{j}^{\prime \prime} u_{k}^{\prime \prime}\right) \frac{\partial \epsilon}{\partial x_{k}}\right] \\
& +\omega\left(C_{\epsilon 1} \frac{1}{2} P_{k k}-C_{\epsilon 2} \bar{\rho} \epsilon\right)+\left\langle\mathcal{S}_{\epsilon}\right\rangle
\end{aligned}
$$

We use the constant values $C_{\epsilon 1}=1.60$ and $C_{\epsilon 2}=1.92$. Note that the two-way coupling source term $\left\langle\mathcal{S}_{\epsilon}\right\rangle$ given by (36) is different than the source term used in [11]. 


\section{Dispersed Phase Modelling}

\subsection{Statistical description of the dispersed phase}

The dispersed phase of the spray is described in terms of the discrete joint mass density function of diameter, velocity and seen velocity (droplet MDF):

$$
\begin{aligned}
\mathcal{F}_{\mathrm{p}} & \left(\boldsymbol{x}, d_{\mathrm{p}}, \boldsymbol{V}_{\mathrm{p}}, \boldsymbol{V}_{\mathrm{s}} ; t\right) \\
& =m_{\mathrm{p}}\left(d_{\mathrm{p}}\right)\left\langle\sum_{+} \delta\left(\boldsymbol{X}_{\mathrm{p}}^{+}-\boldsymbol{x}\right) \cdot \delta\left(D_{\mathrm{p}}^{+}-d_{\mathrm{p}}\right) \cdot \delta\left(\boldsymbol{U}_{\mathrm{p}}^{+}-\boldsymbol{V}_{\mathrm{p}}\right) \cdot \delta\left(\boldsymbol{U}_{\mathrm{s}}^{+}-\boldsymbol{V}_{\mathrm{s}}\right)\right\rangle .
\end{aligned}
$$

$\boldsymbol{X}_{\mathrm{p}}^{+}$is the droplet position vector, $D_{\mathrm{p}}^{+}$the constant droplet diameter, $\boldsymbol{U}_{\mathrm{p}}^{+}$the droplet velocity and $\boldsymbol{U}_{\mathrm{s}}^{+}$the fluid velocity seen by the droplet. The sum in (38) is over the $N_{\mathrm{p}}(t)$ droplets present in the domain at time $t$, such that $\mathcal{F}_{\mathrm{p}}\left(\boldsymbol{x}, d_{\mathrm{p}}, \boldsymbol{V}_{\mathrm{p}}, \boldsymbol{V}_{\mathrm{s}} ; t\right)$.d $d_{\mathrm{p}} . \mathrm{d} \boldsymbol{V}_{\mathrm{p}} . \mathrm{d} \boldsymbol{V}_{\mathrm{s}}$ gives the probable mass of droplets present at $(\boldsymbol{x}, t)$ with diameter in the range $\left[d_{\mathrm{p}}, d_{\mathrm{p}}+\mathrm{d} d_{\mathrm{p}}\right]$, velocity in $\left[\boldsymbol{V}_{\mathrm{p}}, \boldsymbol{V}_{\mathrm{p}}+\mathrm{d} \boldsymbol{V}_{\mathrm{p}}\right]$ and seeing a fluid velocity in $\left[\boldsymbol{V}_{\mathrm{s}}, \boldsymbol{V}_{\mathrm{s}}+\mathrm{d} \boldsymbol{V}_{\mathrm{s}}\right]$.

We define a conditional expectation $\langle\mid\rangle_{\mathrm{p}}$ such that:

$$
\begin{aligned}
& \left\langle Q_{\mathrm{p}}^{+} \mid \boldsymbol{x}, \boldsymbol{V}_{\mathrm{p}}, \boldsymbol{V}_{\mathrm{s}} ; t\right\rangle_{\mathrm{p}} \mathcal{F}_{\mathrm{p}}\left(\boldsymbol{x}, d_{\mathrm{p}}, \boldsymbol{V}_{\mathrm{p}}, \boldsymbol{V}_{\mathrm{s}} ; t\right) \\
& \quad=\left\langle\sum_{+} m_{\mathrm{p}} Q_{\mathrm{p}}^{+} \cdot \delta\left(\boldsymbol{X}_{\mathrm{p}}^{+}-\boldsymbol{x}\right) \cdot \delta\left(D_{\mathrm{p}}^{+}-d_{\mathrm{p}}\right) \cdot \delta\left(\boldsymbol{U}_{\mathrm{p}}^{+}-\boldsymbol{V}_{\mathrm{p}}\right) \cdot \delta\left(\boldsymbol{U}_{\mathrm{s}}^{+}-\boldsymbol{V}_{\mathrm{s}}\right)\right\rangle .
\end{aligned}
$$

In the absence of mass transfer, collisions, coalescence and breakup, the droplet MDF transport equation reads [16]:

$$
\begin{aligned}
\frac{\partial \mathcal{F}_{\mathrm{p}}}{\partial t}+V_{\mathrm{p}, j} \frac{\partial \mathcal{F}_{\mathrm{p}}}{\partial x_{j}}= & -\frac{\partial}{\partial V_{\mathrm{p}, i}}\left[\left\langle\frac{\mathrm{d} U_{\mathrm{p}, i}^{+}}{\mathrm{d} t} \mid \boldsymbol{x}, \boldsymbol{V}_{\mathrm{p}}, \boldsymbol{V}_{\mathrm{s}} ; t\right\rangle_{\mathrm{p}} \mathcal{F}_{\mathrm{p}}\right] \\
& -\frac{\partial}{\partial V_{\mathrm{s}, i}}\left[\left\langle\frac{\mathrm{d} U_{\mathrm{s}, i}^{+}}{\mathrm{d} t} \mid \boldsymbol{x}, \boldsymbol{V}_{\mathrm{p}}, \boldsymbol{V}_{\mathrm{s}} ; t\right\rangle_{\mathrm{p}}\right] .
\end{aligned}
$$

\subsection{Lagrangian modelling of the droplet MDF}

In order to model and solve (40), a particle method is used. A set of uniformly distributed computational droplets (each having a position, diameter, velocity and seen velocity) evolves according to stochastic differential equations such that the ensemble provides a numerical approximation of the modelled droplet $\mathrm{MDF} \mathcal{F}_{\mathrm{p}}^{P}$.

For the non-evaporating spray considered, each computational droplet has a set of properties $\left\{n_{\mathrm{p}}^{*}, \boldsymbol{X}_{\mathrm{p}}^{*}, D_{\mathrm{p}}^{*}, \boldsymbol{U}_{\mathrm{p}}^{*}, \boldsymbol{U}_{\mathrm{s}}^{*}\right\}$, where $n_{\mathrm{p}}^{*}$ is a weight factor associated to the particle. ${ }^{2}$

\footnotetext{
${ }^{2}$ A computational droplet is not in one to one correspondence to a "real" droplet: each computational droplet is a statistical sample of the dispersed phase. The weight factors accommodate the difference between number of samples and the number of real droplets.
} 
The superscript $*$ denotes that the quantity is a stochastic particle property. The modelled droplet MDF is defined as

$$
\begin{aligned}
\mathcal{F}_{\mathrm{p}}^{P} & \left(\boldsymbol{x}, d_{\mathrm{p}}, \boldsymbol{V}_{\mathrm{p}}, \boldsymbol{V}_{\mathrm{s}} ; t\right) \\
& =\left\langle\sum_{*} n_{\mathrm{p}}^{*} m_{\mathrm{p}}^{*} \cdot \delta\left(\boldsymbol{X}_{\mathrm{p}}^{*}(t)-\boldsymbol{x}\right) \cdot \delta\left(D_{\mathrm{p}}^{*}-d_{\mathrm{p}}\right) \cdot \delta\left(\boldsymbol{U}_{\mathrm{p}}^{*}(t)-\boldsymbol{V}_{\mathrm{p}}\right) \cdot \delta\left(\boldsymbol{U}_{\mathrm{s}}^{*}(t)-\boldsymbol{V}_{\mathrm{s}}\right)\right\rangle,
\end{aligned}
$$

where $m_{\mathrm{p}}^{*}=\rho_{\mathrm{p}} \pi\left(D_{\mathrm{p}}^{*}\right)^{3} / 6$ is the mass of the computational droplet (assumed to be spherical). Unconditional droplet mean properties in a small domain $\Omega$ of volume $\mathcal{V}_{\Omega}$, are obtained as:

$$
\rho_{\mathrm{p}}\left\langle Q_{\mathrm{p}}\right\rangle=\frac{1}{\mathcal{V}_{\Omega}}\left\{\sum_{* \text { in } \Omega} m_{\mathrm{p}}^{*} Q_{\mathrm{p}}^{*}(t)\right\}_{T A},
$$

and conditional averages are obtained as:

$$
\left\langle Q_{\mathrm{p}}\right\rangle_{\mid \mathrm{p}}=\left\{\sum_{* \text { in } \Omega} m_{\mathrm{p}}^{*} Q_{\mathrm{p}}^{*}(t)\right\}_{T A} /\left\{\sum_{* \text { in } \Omega} m_{\mathrm{p}}^{*}\right\}_{T A},
$$

where \{\}$_{T A}$ is an iteration averaging operator [17].

\subsection{Dispersion model}

The stochastic particle position $\boldsymbol{X}_{\mathrm{p}}^{*}$ and velocity $\boldsymbol{U}_{\mathrm{p}}^{*}$ follow the simplified equations of motion (4) and (5).

A Langevin model is used to model increments of the seen velocity $\boldsymbol{U}_{\mathrm{s}}$ over small time steps $\mathrm{d} t$. The model of Minier and Peirano [13], written for the fluctuating seen velocity, $\boldsymbol{u}_{\mathrm{s}}^{\prime \prime}=\boldsymbol{U}_{\mathrm{s}}-\widetilde{\boldsymbol{U}},[16]$ reads:

$$
\begin{aligned}
d u_{\mathrm{s}, i}^{\prime \prime}= & -\left(U_{\mathrm{p}, j}-\left\langle U_{\mathrm{p}, j}\right\rangle_{\mid \mathrm{p}}+\left\langle u_{\mathrm{s}, j}^{\prime \prime}\right\rangle_{\mid \mathrm{p}}\right) \frac{\partial \widetilde{U}_{i}}{\partial x_{j}} d t+\frac{1}{\bar{\rho}} \frac{\partial}{\partial x_{j}}\left(\bar{\rho} \widetilde{u}_{i}^{\pi \prime} u_{j}^{\prime \prime}\right) d t \\
& +G_{\mathrm{s}, i j} u_{\mathrm{s}, j}^{\prime \prime} d t+B_{\mathrm{s}, i j} d W_{j} .
\end{aligned}
$$

where $W_{j}(t)$ are independent Wiener processes $\left(\mathrm{d} W_{j}\right.$ is a Gaussian stochastic process of zero mean and variance $\mathrm{d} t)$. The matrices $G_{\mathrm{s}, i j}$ and $B_{\mathrm{s}, i j}$ [13] are functions of Reynolds stresses $u_{i}^{\prime \prime} u_{j}^{\prime \prime}$, dissipation $\epsilon$ and mean drift velocity $\left\langle\boldsymbol{U}_{\mathrm{r}}\right\rangle_{\mid \mathrm{p}}=\left\langle\boldsymbol{U}_{\mathrm{p}}-\boldsymbol{U}_{\mathrm{s}}\right\rangle_{\mid \mathrm{p}}$. For polydispersed flows, (44) is written for each size class. This means that the conditional averages \langle\rangle$_{\mid \mathrm{p}}$ are actually evaluated for each size class separately (class averages) [17].

In the limit of tracer particles, $\left\langle\boldsymbol{U}_{\mathrm{r}}\right\rangle_{\mid \mathrm{p}}$ tends to zero and $G_{\mathrm{s}, i j}$ and $B_{\mathrm{s}, i j}$ tend to the coefficient of the Simplified Langevin Model, implying a Eulerian evolution of the Reynolds stresses corresponding to the Rotta model. Hence there is a small inconsistency if the LRR Reynolds stress model is used for the continuous phase modelling. Moreover we do not account for the two-way coupling source terms in the seen velocity fluctuation increments (44): $\Pi_{i j}^{(\mathrm{fp})}$ and $\left\langle\mathcal{S}_{u_{i} u_{j}}\right\rangle$ are not taken into account. However, the focus of this paper is on the two-way coupling source terms in the continuous phase and the details of the dispersion model will not affect the conclusions of this work. 


\section{Application to a Turbulent Two-phase Jet: Configuration and Boundary Conditions}

To investigate the importance of including the two-way coupling source terms in the model for the pressure rate of strain, simulations of a turbulent polydispersed non-evaporating spray were performed. The configuration corresponds to the spray investigated experimentally in $[3,4]$. The experiment is characterised as a constant density air jet laden with liquid particles flowing downwards, issuing from a $8 \mathrm{~mm}$ diameter nozzle into a cubic chamber with sides of $0.5 \mathrm{~m}$. The sides are far away from the edge of the spray and the influence of the presence of the wall on the spray behaviour can be neglected.

In this experiment, a two-component phase Doppler anemometer (PDA) was used to simultaneously measure droplet velocities and diameters and laser induced fluorescence (LIF) combined with PDA was used to extract the local value of concentration and flux per droplet size class. The estimated statistical errors differ per size class, ranging from $0.8 \%$ for the smallest size class $(0-5 \mu \mathrm{m})$ to $7.3 \%$ for the size class $80-90 \mu \mathrm{m}$. This effect is mainly due to a decreasing number of samples for increasing droplet size class. Four cases were investigated experimentally with different liquid mass loadings, i.e. $\phi=0$ (gas flow only), $\phi=0.12, \phi=0.41$ and $\phi=0.73$. We will discuss results for the test-case with the highest mass loading only. The gas-particle interactions are the strongest for this test-case and will therefore illustrate the importance between the different two-way coupling terms more clearly.

The droplet sizes range from 1-120 m. The droplets in the smallest size class are assumed to behave as tracer particles and can be used as an estimation for the gasphase mean and rms velocity. The inlet conditions for the simulation correspond to the measured values in the section closest to the nozzle, at $x=20 \mathrm{~mm}$ downstream. The inlet boundary data not available experimentally is the profile for turbulent dissipation. This profile was obtained by first estimating the dissipation profile for

Fig. 1 Experimental setup and computational domain

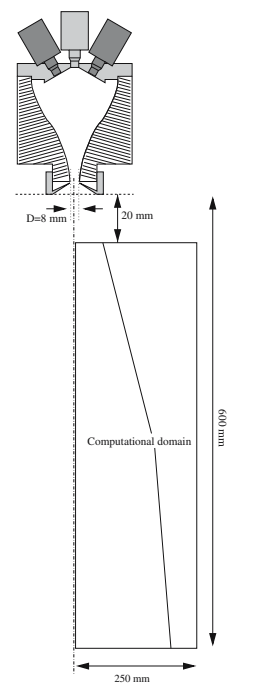

\begin{tabular}{ll}
\hline Re & 9500 \\
$\phi$ & 0.73 \\
$\rho_{p}$ & $760 \mathrm{~kg} / \mathrm{m}^{3}$ \\
domain & $600 \times 250$ \\
grid cells & $120 \times 64$ on a stretched grid \\
\hline
\end{tabular}


the unladen jet case, and then adding an extra dissipation term $\frac{1}{2}\left\langle\mathcal{S}_{u_{k}^{\prime \prime} u_{k}^{\prime \prime}}\right\rangle$ due to twoway coupling.

A sketch of the experimental setup together with a table with some additional relevant information is shown in Fig. 1. The Cartesian grid used for the computations consists of 120 cells in axial direction and 64 cells in the radial direction. A local time-stepping algorithm for the gas phase as well as for the dispersed phase was used to determine the time step used in each cell $[15,17]$. For the dispersed phase, five particles per class per cell were used and 13 droplet classes were considered. An iteration averaging scheme [16] was used to reduce the statistical error, which allowed us to use this very low value for the number of particles per class per cell.

\section{Results and Discussion}

Four simulations were performed to investigate the influence of the different twoway coupling terms:

(a) A simulation without any two-way coupling was performed as a reference.

(b) A simulation taking into account only the two-way coupling effects in the transport equation for momentum.

(c) A simulation taking into account the two-way coupling effects in the transport equation for momentum, dissipation and Reynolds stresses (called full coupling in the legend).

(d) A simulation similar to the previous simulation (full coupling), but additionally taking into account the source term appearing in the model for the pressure rate of strain.

The numerical results at four axial locations downstream of the injector will be discussed, i.e. at $x=40, x=80, x=160$ and $x=320 \mathrm{~mm}$ downstream of the injector, which corresponds to 5, 10, 20 and 40 injector diameters.

\subsection{Results for the dispersed phase}

We will now first discuss some numerical results for the dispersed phase. In the simulation results shown, all two-way coupling source terms were taken into account (case $(d)$, including the extra source terms in the model for the pressure rate of strain). Figure 2 shows the mean axial velocity of the dispersed phase $U_{p}$ for three droplet size classes at four locations downstream from the injector. The axial velocity is only slightly underpredicted, and the agreement with the experimental data is very good. The Reynolds stresses $\sqrt{u_{p} u_{p}}$ and $\sqrt{v_{p} v_{p}}$ are shown in Figs. 3 and 4 respectively. The Reynolds stresses of the dispersed phase are slightly overpredicted in the first two downstream locations. Further downstream, the Reynolds stresses $\sqrt{u_{p} u_{p}}$ are slightly underpredicted, but we still overpredict the value of $\sqrt{v_{p} v_{p}}$. However, the discrepancy is not very large and there is still a good agreement with the experimental data.

The size classes shown in the figures are the size classes 40-50, 70-80 and 100$110 \mu \mathrm{m}$. The size classes between 40-100 $\mu \mathrm{m}$ contribute the most to the two-way coupling source term. The larger size classes will also be less influenced by changes in the continuous phase, and the different two-way coupling models will have only a 

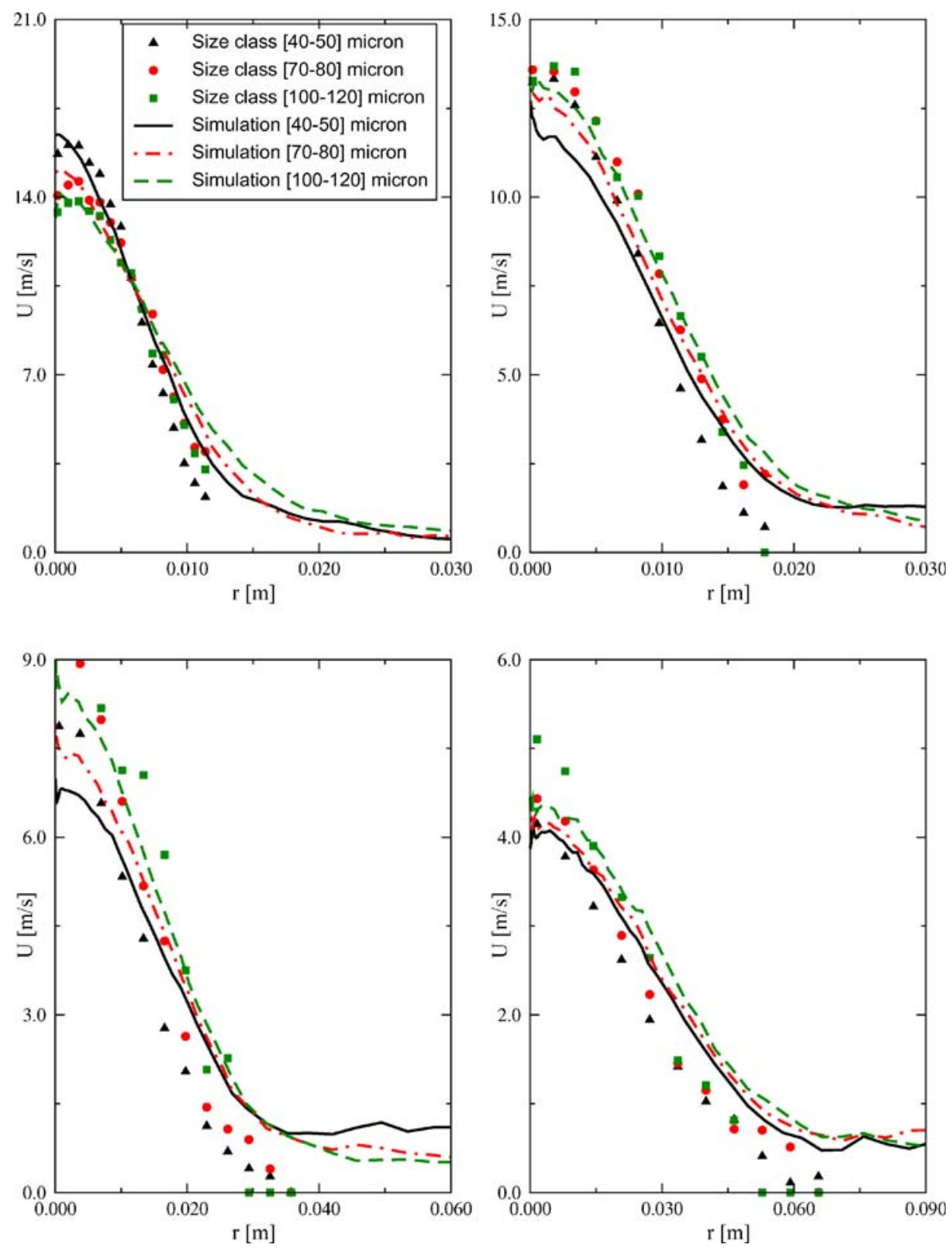

Fig. 2 Axial velocity of the dispersed phase at locations $x=40, x=80, x=160, x=320 \mathrm{~mm}$ downstream of the inlet for case $(d)$

small effect on the dispersed-phase properties. The velocity of the smallest droplet size class will quickly relax to the velocity of the continuous phase and the particles will then more or less follow the fluid flow. This size class will be affected the most by changes in the continuous phase, but these small particles will also have a negligible contribution to the two-way coupling source term. Since the largest droplet classes 

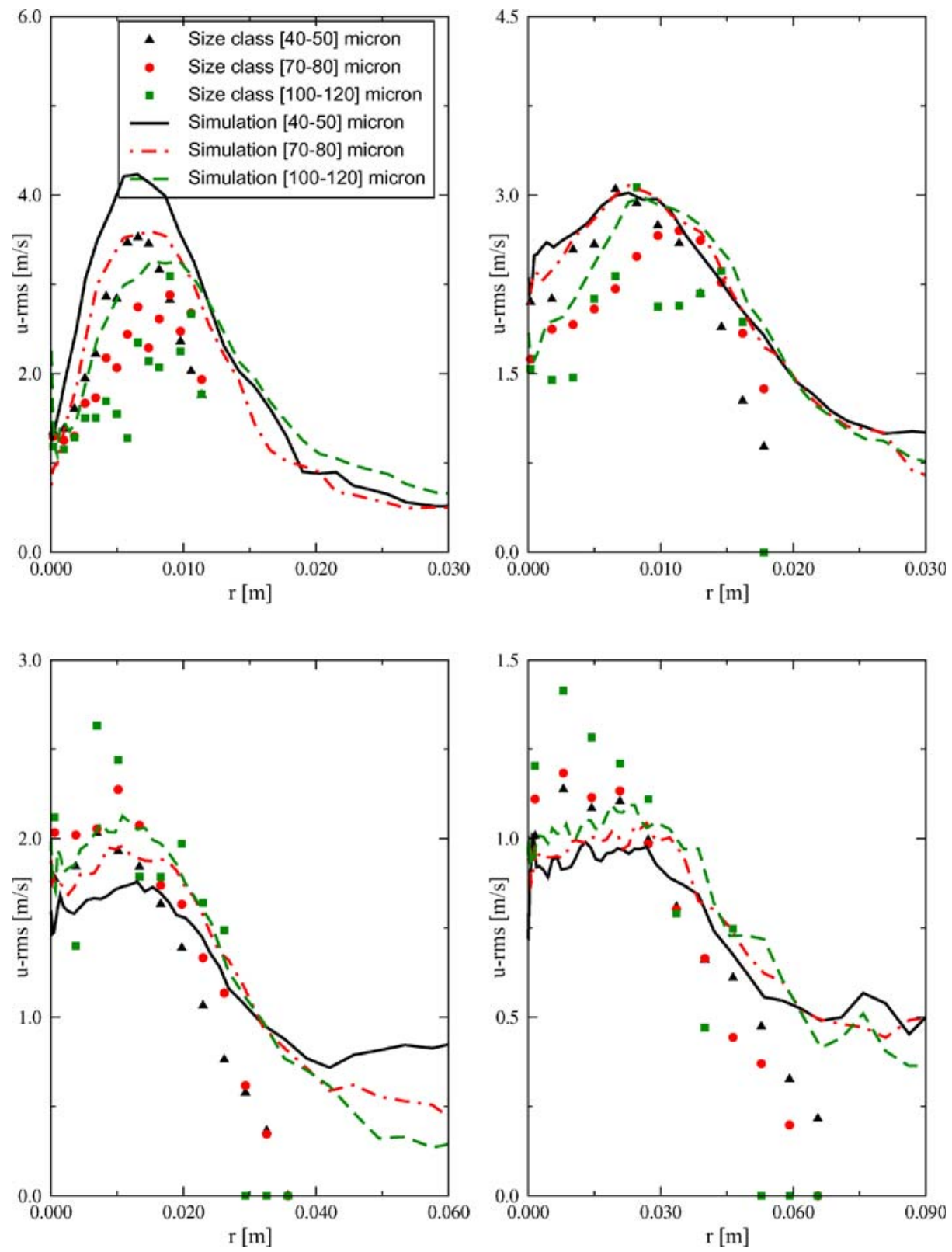

Fig. 3 Reynolds stress $\sqrt{u^{\prime \prime} p u^{\prime \prime} p}$ at locations $x=40, x=80, x=160, x=320 \mathrm{~mm}$ downstream of the inlet for case $(d)$

are the least affected by the surrounding gas flow, the discrepancy between the experimental and numerical results for the dispersed-phase Reynolds stresses may well be caused by modelling issues in the dispersed phase only. Since the performance analysis of the dispersion model is not the focus of this article, we will therefore focus our analysis on the results of the continuous phase. 

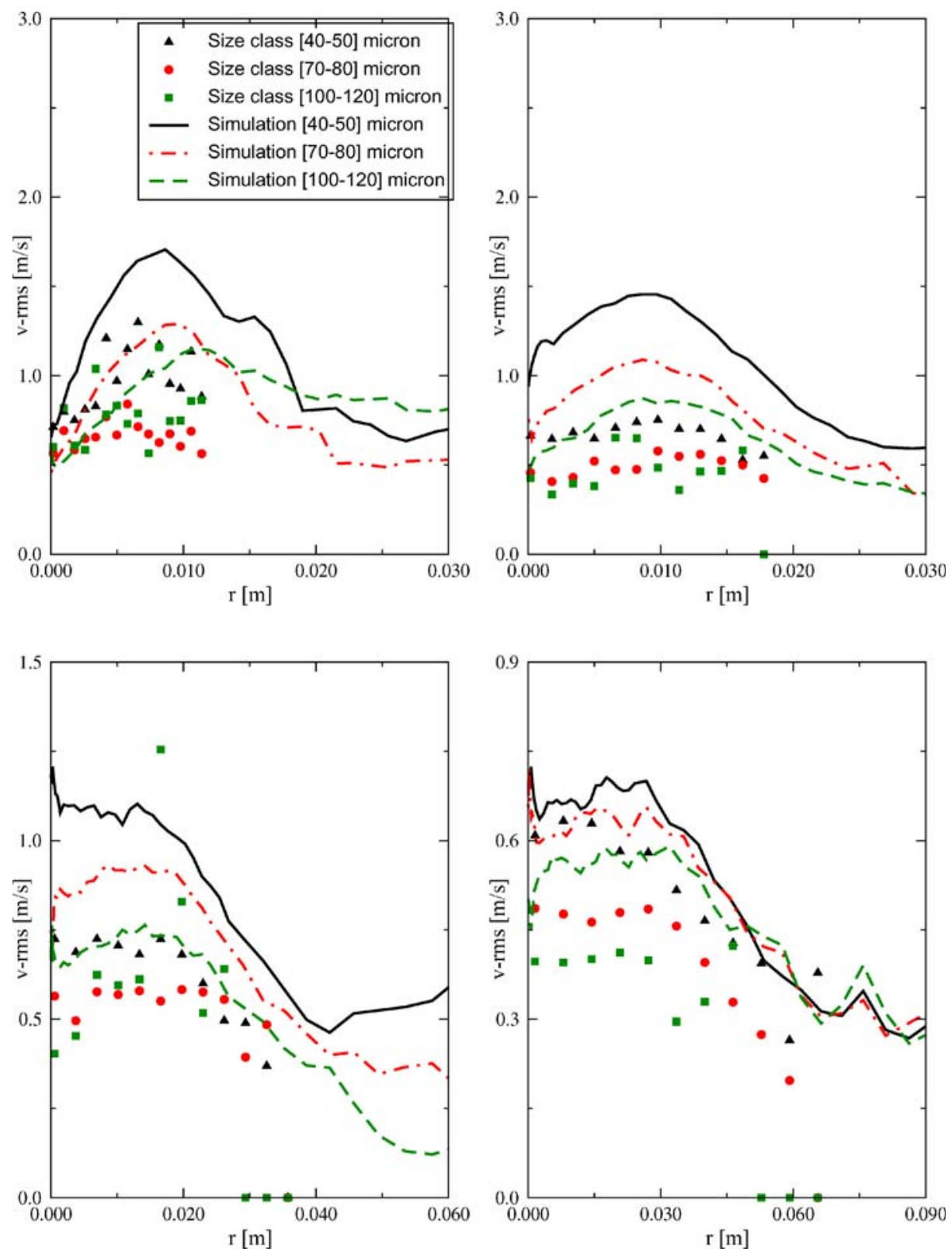

Fig. 4 Reynolds stress $\sqrt{v^{\prime \prime} p v^{\prime \prime} p}$ at locations $x=40, x=80, x=160, x=320 \mathrm{~mm}$ downstream of the inlet for case $(d)$

\subsection{Results for the continuous phase}

Figure 5 shows the axial velocity of the continuous phase for the four different simulations. For comparison, the experimental results of the single-phase test case was added to the graph as well. In the simulation without two-way coupling, the 

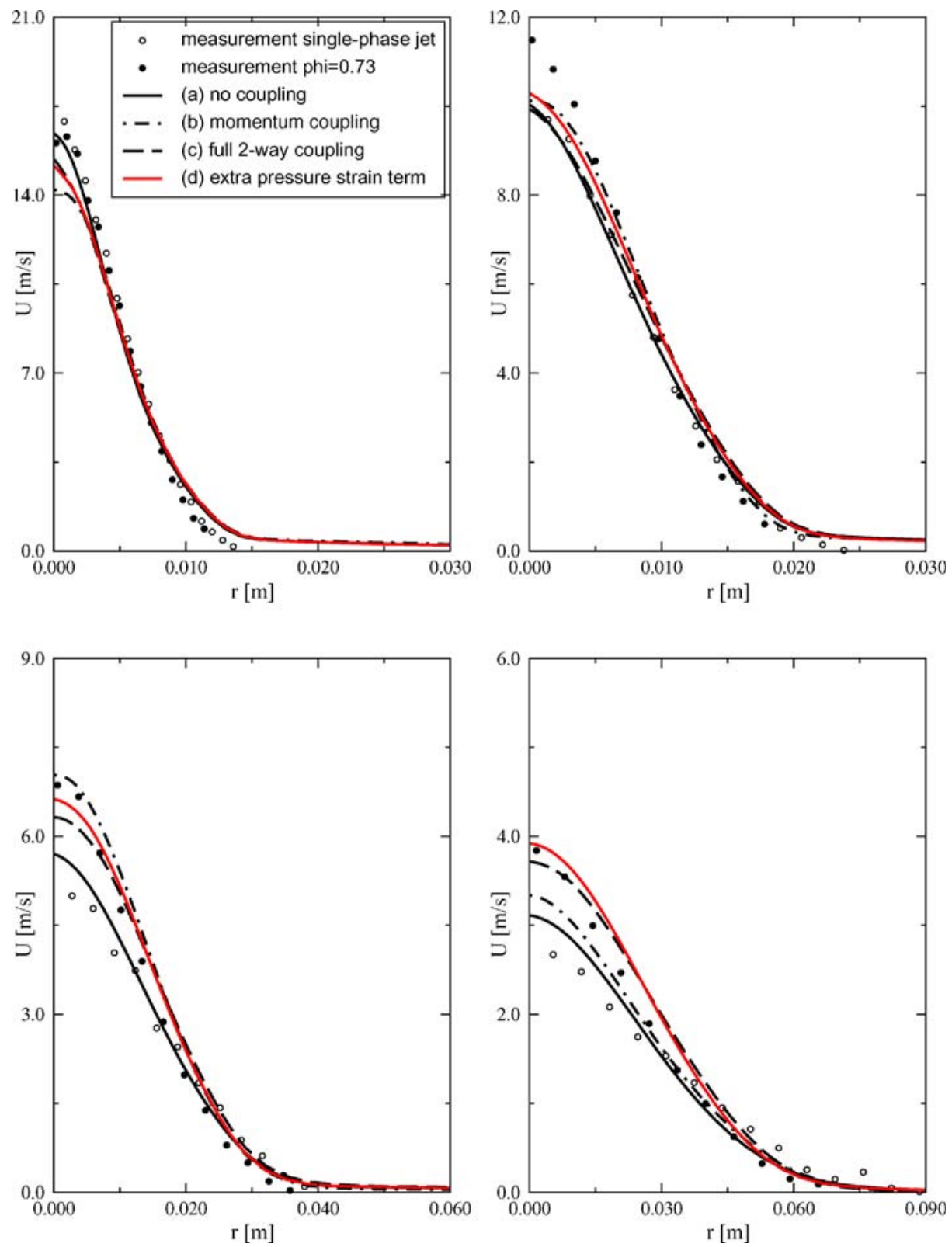

Fig. 5 Axial velocity of the gas phase at locations $x=40, x=80, x=160, x=320 \mathrm{~mm}$ downstream of the inlet

continuous phase is not influenced by the presence of the particles. This axial velocity profile quickly relaxes to the experimentally measured axial velocity profile of the single-phase flow. Only adding the two-way coupling source terms in the momentum equation is not sufficient to get a good correspondence with the measurements. When taking into account the two-way coupling source terms in the Reynolds stress 
equations and the dissipation equation (full coupling), the axial velocity downstream of the injector is now slightly underpredicted. When taking into account the extra particle source terms in the model for the pressure rate of strain, the prediction of the axial velocity of the gas phase improves slightly.
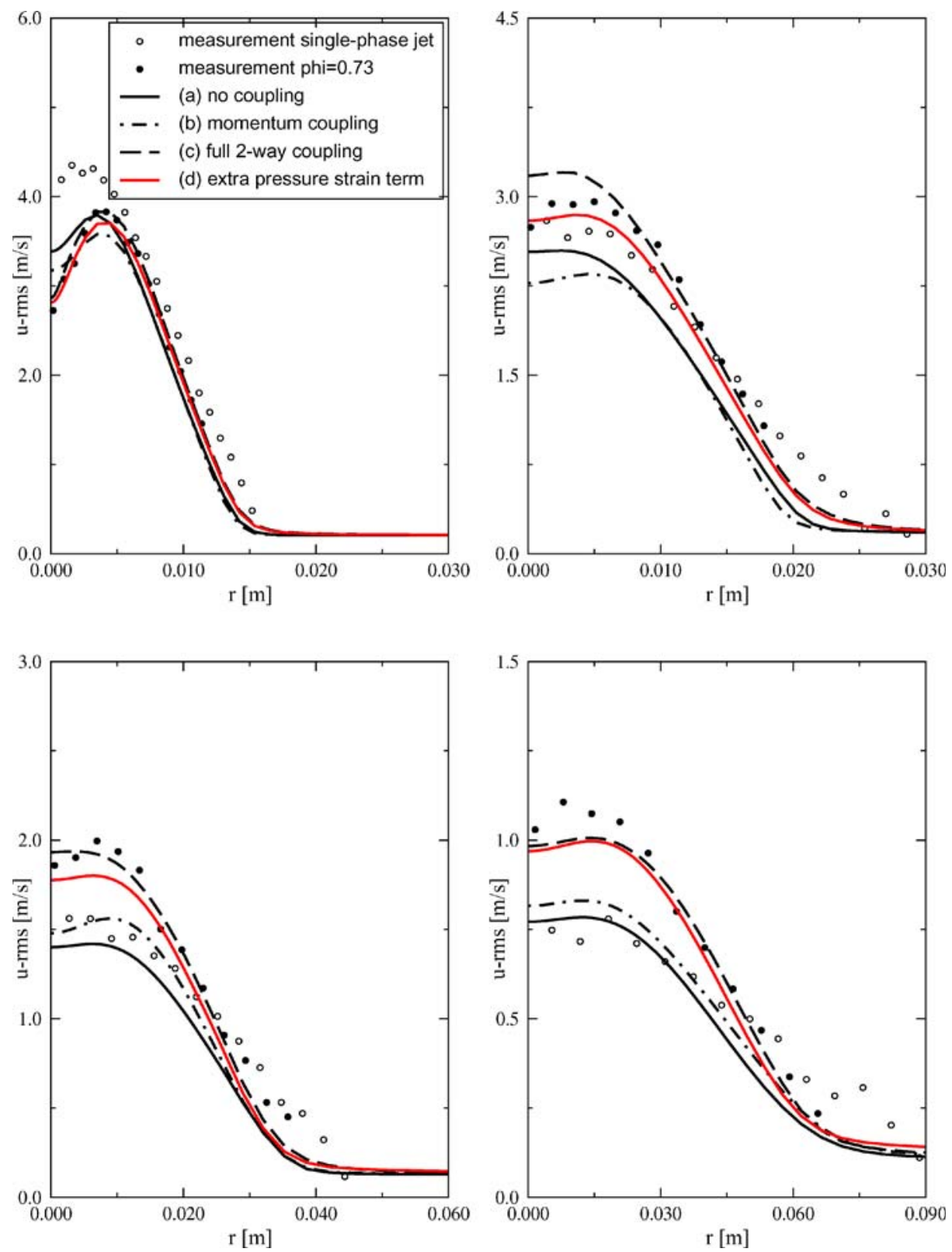

Fig. 6 Reynolds stress $\sqrt{u^{\prime \prime} u^{\prime \prime}}$ at locations $x=40, x=80, x=160, x=320 \mathrm{~mm}$ downstream of the inlet 
Larger difference can be observed in the profiles for the normal Reynolds stresses $\sqrt{\widetilde{u u}}$ and $\sqrt{\widetilde{v v}}$, shown in Figs. 6 and 7 respectively. We can again see that without two-way coupling the Reynolds stress profiles relax to the experimental values of the single-phase jet. Adding two-way coupling in the momentum equation only is
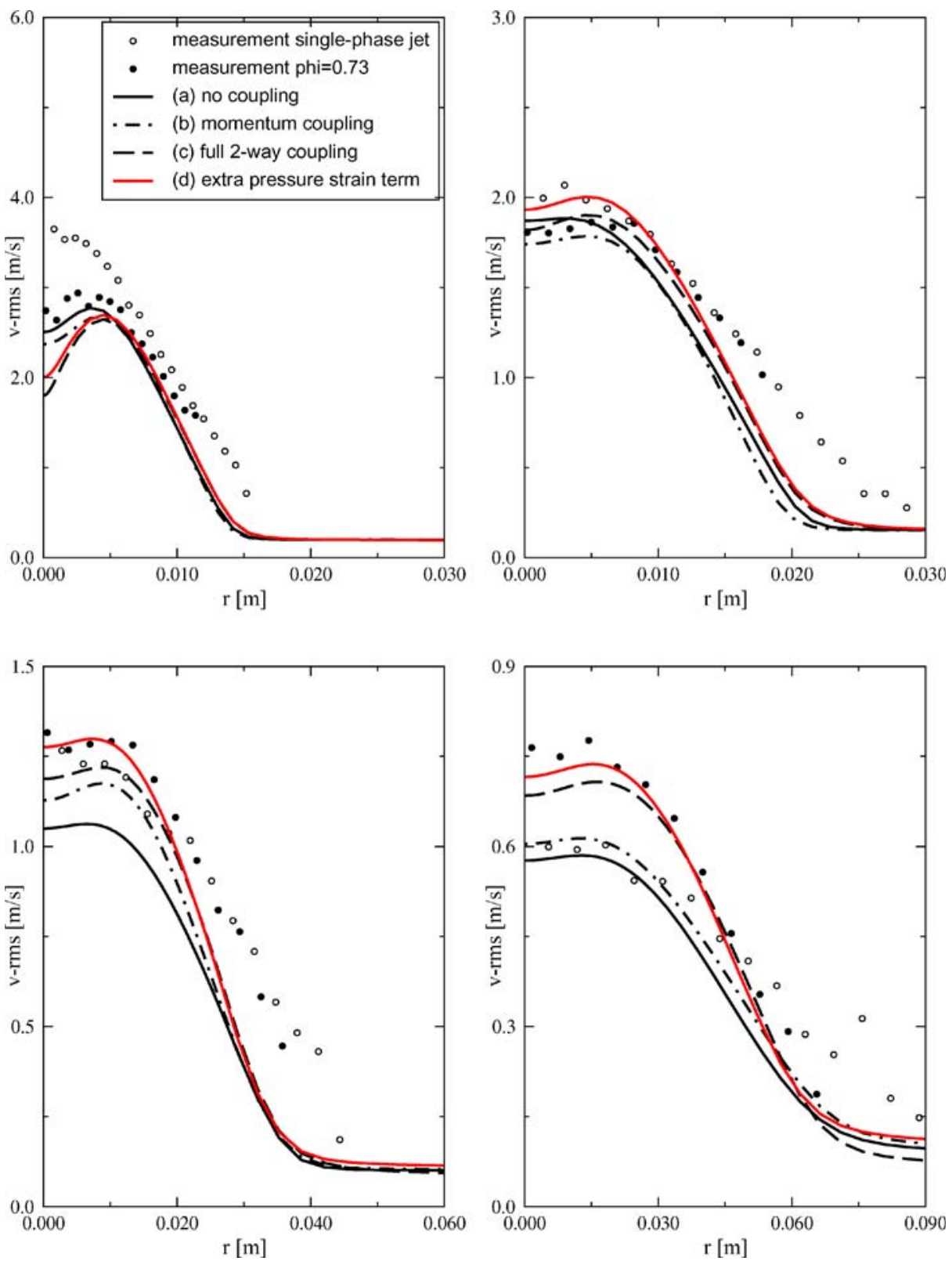

Fig. 7 Reynolds stress $\sqrt{v^{\prime \prime} v^{\prime \prime}}$ at locations $x=40, x=80, x=160, x=320 \mathrm{~mm}$ downstream of the inlet

Springer 
not sufficient to improve the predictions of the Reynolds stresses, but full twoway coupling improves the results at all downstream locations. The performance difference between model $(c)$ and model $(d)$ is not clear from Figs. 6 and 7, but model $(d)$ tends to decrease the Reynolds stress anisotropy compared to model $(c)$.
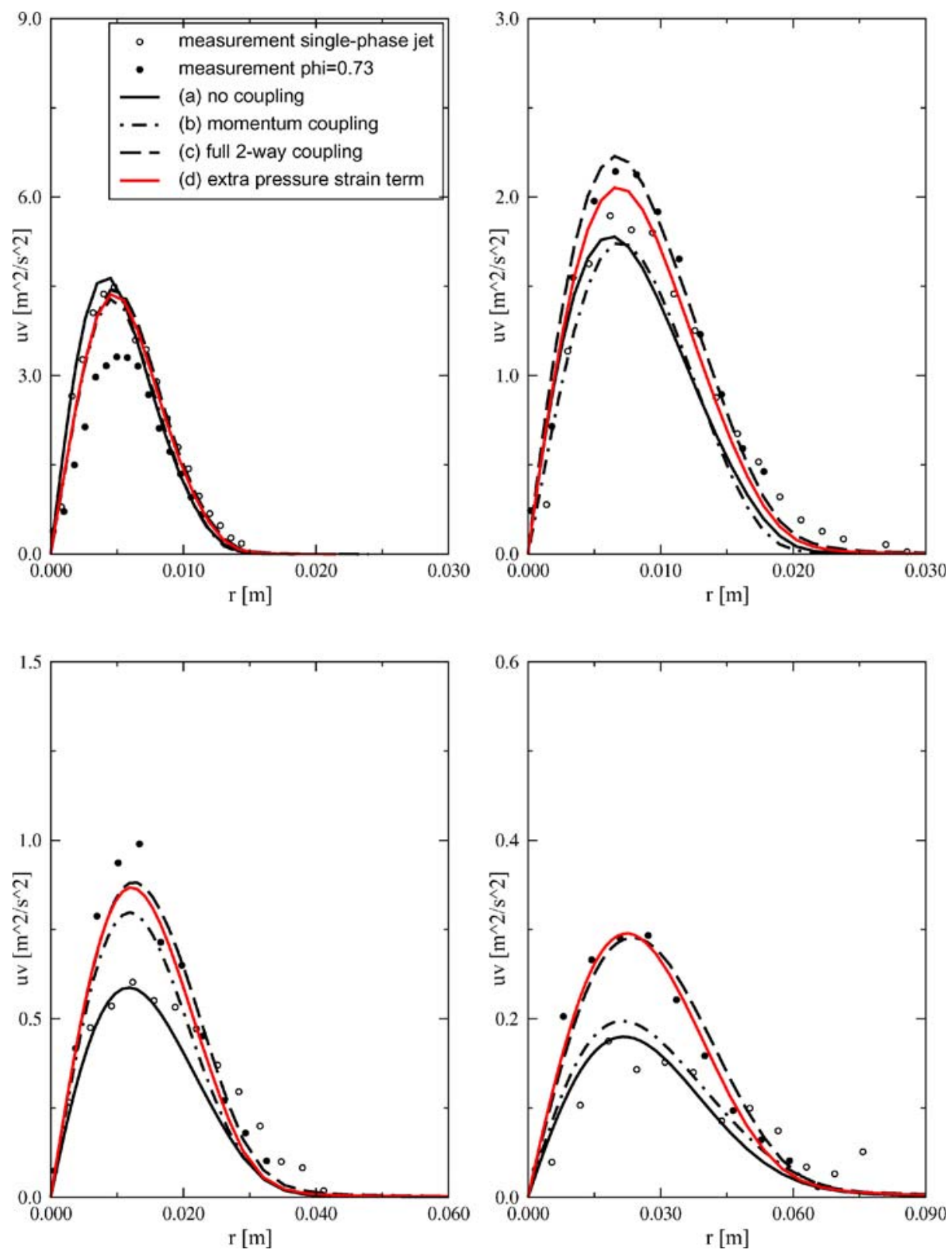

Fig. 8 Reynolds stress $\sqrt{u^{\prime \prime} v^{\prime \prime}}$ at locations $x=40, x=80, x=160, x=320 \mathrm{~mm}$ downstream of the inlet 
Further downstream the effect of the source term in the pressure rate of strain decreases (as the turbulence becomes more isotropic) and the difference between simulations $(c)$ and $(d)$ becomes smaller. For the Reynolds stress $\sqrt{\widetilde{v v}}$ shown in Fig. 7 the experimentally measured profile at $x=40 \mathrm{~mm}$ has higher values at the symmetry axis than $\sqrt{\widetilde{u u}}$. When no two-way coupling effects are present, the experimental value of $\sqrt{\widetilde{v v}}$ is higher than that of the experiment with $\phi=0.73$ in this figure. The twoway coupling effects cause a reduction of the Reynolds stress $\sqrt{\widetilde{v v}}$. The simulation with no coupling also predicts higher values of $\sqrt{\widetilde{v v}}$ than the simulations with twoway coupling. However, models $(c)$ and $(d)$ underpredict the value of the Reynolds stress. This could indicate an overprediction of the magnitude of the source term for the transport equation of $\sqrt{\widetilde{v v}}$. Further downstream we see that both models $(c)$ and (d) are able to capture the correct behaviour of $\sqrt{\widetilde{v v}}$ and there is good agreement with the experimental results. The values for the shear stress $\sqrt{\widetilde{u v}}$, which is shown in Fig. 8, are overpredicted near the injector, but further downstream there is good agreement between the experimental data and the predictions from the simulations for all three two-way coupling models.

\section{Conclusions}

In this work, we have derived a modified Reynolds stress model for dispersed twophase flows that takes into account the modification of the pressure rate of strain due to the presence of the dispersed phase. The extra two-way coupling source terms appearing in the model for the pressure rate of strain remain relatively simple and can be described with a model containing only two constants. When the second model constant is set to zero, the model reduces to a form similar to the Rotta model for single-phase flows, but with an anisotropy tensor based on the Reynolds stress two-way coupling source term. A simulation of a polydispersed two-phase flow was performed to investigate the importance of the different two-way coupling terms appearing in the Reynolds averaged transport equations. For this particular test case, taking into account the particle source term in the model for the pressure rate of strain leads to a small improvement of the predictions of the gas-phase properties. The importance of this source term will however become more significant in flows with a higher fluid-particle anisotropy. The determination of more optimal constants for the source term appearing in the model for the pressure rate of strain, is left for future research.

Acknowledgements This work was financially supported by the Technology Foundation STW. Part of the work has been financed by the COMLIMANS program and by the Spanish MEC under Project ENE 2005-09190-C04-04/CON.

\section{References}

1. Crowe, C.T.: On models for turbulence modulation in fluid-particle flows. Int. J. Multiph. Flow 26, 719-727 (2000)

2. Drew, D.A.: Mathematical modeling of two-phase flow. Annu. Rev. Fluid Mech. 15, 261-291 (1983) 
3. Ferrand, V., Bazile, R., Boree, J.: Measurements of concentration per size class in a dense polydispersed jet using planar laser-induced fluorescence and phase Doppler techniques. Exp. Fluids 31, 597-607 (2001)

4. Ferrand, V., Bazile, R., Boree, J., Charnay, G.: Gas-droplet turbulent velocity correlations and two-phase interaction in an axisymmetric jet laden with partly responsive droplets. Int. J. Multiph. Flow 29, 195-217 (2003)

5. Gatignol, R.: The Faxén formulae for a rigid particle in an unsteady non-uniform Stokes flow. J. Méc. Théor. Appl 1, 143-160 (1983)

6. Gouesbet, G., Berlemont, A.: Eulerian and Lagrangian approaches for predicting the behaviour of discrete particles in turbulent flows. Pror. Energy Combust. Sci. 25, 133-159 (1999)

7. Hishida, K., Maeda, M.: Turbulent characteristics of gas-solids two-phase confined jet: effect of particle density. Japanese Journal of Multiphase Flow 1, 56-69 (1987)

8. Kataoka, I.: Local instant formulation of two-phase flow. Int. J. Multiph. Flow 12, 745-758 (1986)

9. Lain, S., Sommerfeld, M.: Turbulence modulation in dispersed two-phase flow laden with solids from a Lagrangian perspective. Int. J. Heat Fluid Flow 24, 616-625 (2003)

10. Launder, B.E., Reece, G.J., Rodi, W.: Progress in the development of a Reynolds-stress turbulence closure. J. Fluid Mech. 68, 537-566 (1975)

11. Mashayek, F., Taulbee, D.B.: Turbulent gas-solid flows part I: direct simulations and Reynolds stress closures. Numer. Heat Transf. B 41, 1-29 (2002)

12. Maxey, M.R., Riley, J.J.: Equation of motion for a small rigid sphere in a nonuniform flow. Phys. Fluids 26, 883-889 (1983)

13. Minier, J.P., Peirano, E.: The PDF approach to turbulent polydispersed two-phase flows. Phys. Rep. 352, 1-214 (2001)

14. Mostafa, A.A., Mongia, H.C., McDonell, V.G., Samuelsen, G.S.: An experimental and numerical study of particle-laden coaxial jet flows. Int. J. Heat Fluid Flow 11, $90-97$ (1990)

15. Muradoğlu, M., Pope, S.B.: A local time-stepping algorithm for solving the probability density function turbulence model equations. AIAA J. 40, 1755-1763 (2002)

16. Naud, B.: PDF modeling of turbulent sprays and flames using a particle stochastic approach. Ph.D. thesis, Delft University of Technology. Downloadable from http://www.darenet.nl/ promiseofscience (2003)

17. Naud, B., Jiménez, C., Roekaerts, D.: Velocity-position error for tracer particles in stochastic Lagrangian modelling of polydispersed sprays based on the seen velocity. In: 5th International Conference on Multiphase Flow, ICMF'04 (2004)

18. Simonin, O.: Statistical and continuum modeling of turbulent reactive flows - part I. Lecture series 2000-06 of the von Karman Institute: Theoretical and Experimental Modelling of Particulate Flow, 3-7 April 2000

19. Simonin, O., Deutsch, E., Minier, J.P.: Eulerian prediction of the fluid/particle correlated motion in turbulent two-phase flows. Appl. Sci. Res. 51, 275-283 (1993)

20. Sommerfeld, M., Qiu, H.H.: Experimental studies of spray evaporation in turbulent flow. Int. J. Heat Fluid Flow 19, 10-22 (1998)

21. Speziale, C.G., Sarkar, S., Gatski, T.B.: Modelling the pressure-strain correlation of turbulence: an invariant dynamical systems approach. J. Fluid Mech. 227, 245-272 (1991)

22. Taulbee, D.B., Mashayek, F., Barré, C.: Simulation and Reynolds stress modeling of particleladen turbulent shear flows. Int. J. Heat Fluid Flow 20, 368-373 (1999) 